\title{
Fruit Fly (Diptera: Tephritidae) Fauna of Ordu (Turkey) Province
}

\author{
Murat Kütük ${ }^{1, a, *}$, Ümit Katrancı ${ }^{1, b}$ \\ ${ }^{1}$ Department of Biology, Faculty of Science, Gaziantep University, 27410 Gaziantep, Turkey \\ ${ }^{*}$ Corresponding author

\begin{tabular}{l|l}
\hline A R T I C L E IN F O & A B S T R A C T \\
\hline Research Article & $\begin{array}{l}\text { Fruit flies (Diptera: Tephritidae) are one of the most populous families of Diptera. In this study, it } \\
\text { was aimed to determine the fauna of fruit flies in Ordu province. Adult specimens were collected } \\
\text { from the research region in 2016-2017 years. All the specimens were identified by examining their } \\
\text { morphological characters in Gaziantep University, Biology Department, Entomology Laboratory. As } \\
\text { a result, thirty-three species belonging to 11 genera from three subfamilies were identified. All species } \\
\text { are recorded for the first time from Ordu province. Material examined and wing photographs of the } \\
\text { species are presented. }\end{array}$ \\
$\begin{array}{l}\text { Received : 24/04/2021 } \\
\text { Accepted : } 18 / 05 / 2021\end{array}$
\end{tabular}

Keywords:

Tephritidae

Fruit flies

Fauna

Ordu

Turkey

Türk Tarım - Gıda Bilim Ve Teknoloji Dergisi, 9(6): 1159-1164, 2021

\section{Ordu (Türkiye) İli Meyve Sinekleri (Diptera: Tephritidae) Faunası}

\begin{tabular}{|c|c|}
\hline M A K A L E B İ L G İ S İ & Ö Z \\
\hline $\begin{array}{l}\text { Araştırma } \text { Makalesi } \\
\text { Geliş } \quad: \text { 24/04/2021 } \\
\text { Kabul } \quad: 18 / 05 / 2021\end{array}$ & $\begin{array}{l}\text { Meyve sinekleri (Diptera: Tephritidae), Diptera'nın en kalabalık familyalarından biridir. Bu } \\
\text { çalışmada, Ordu ilindeki meyve sineklerinin faunasının belirlenmesi amaçlanmıştır. Araştırma } \\
\text { bölgesinden 2016-2017 yıllarında ergin örnekler toplanmıştır. Tüm örnekler, Gaziantep Üniversitesi, } \\
\text { Biyoloji Bölümü, Entomoloji Laboratuvarı'nda morfolojik karakterleri incelenerek tanımlandı. Sonuç } \\
\text { olarak, üç alt aileden } 11 \text { cinse ait } 33 \text { tür belirlendi. Tüm türler Ordu ilinden ilk defa kayıt altına } \\
\text { alınmıştır. Türlere ait incelenen materyal ve kanat fotoğrafları sunulmuştur. }\end{array}$ \\
\hline $\begin{array}{l}\text { Anahtar Kelimeler: } \\
\text { Tephritidae } \\
\text { Meyve sinekleri } \\
\text { Fauna } \\
\text { Ordu } \\
\text { Türkiye }\end{array}$ & \\
\hline
\end{tabular}




\section{Introduction}

Fruit flies (Diptera: Tephritidae) mostly host and feed on wild plants belonging to the Asteraceae family (Freidberg and Kugler, 1989). Fruit flies are one of the most populous families of Diptera. It is represented worldwide with 4716 species belonging to 492 genera (Pape et al., 2011). This number has increased with the new species defined in recent years. It has been reported that approximately 1500 of these species related to fruits. In addition, approximately 250 species are known to be economically harmful (Li et al., 2013).

Research on fruit flies fauna in Turkey continues nearly a century. However, the most important contributions have been made in recent years. The last two decades have been identified only 14 new fruit fly species (Tephritis merzi Freidberg \& Kütük, 2002; Urophora doganlari Kütük, 2006; Myoleja korneyevi Han \& Kütük, 2006; Tephritis erdemlii Kütük, 2008; Urophora hani Kütük, 2009a; Terellia yukseli Kütük, 2009b; Terellia askaleensis Kütük et al., 2011; Tephritis ozaslani Kütük et al., 2012; Terellia freidbergi Korneyev et al., 2013; Urophora turkeyensis Yaran \& Kütük, 2014; Carpomya liat Freidberg, 2016; Terellia akguli Yaran et al., 2018; Tephritis turkeri Kütük \& Yaran, 2020; Tephritis kutuki Yaran \& Görmez, 2020) from Turkey. Moreover, the 75-fruit fly has been also reported as new records for Turkey (Kütük, 2003a, 2003b;
Kütük \& Özgür, 2003a, 2003b, 2004; Özgür \& Kütük, 2003, 2005, 2006; Kütük \& Özaslan, 2006; Kütük \& Varol, 2006; Pakyürek, 2006; Görmez, 2011; Bayrak, 2011; Kütük et al., 2013; Yaran \& Kütük, 2015, 2016; Yaran et al., 2018a, 2018b; Çalışkan Keçe et al., 2019; Görmez \& Kütük, 2020; Kütük \& Yaran, 2020). With all these data, the fruit fly fauna of Turkey has reached 173 species.

In this study, it was aimed to determine the fauna of Tephritidae in Ordu province.

\section{Material and Method}

As research region, Ordu province, located in the north of Turkey, was designated (Map 1). The region is largely under the influence of the Black Sea climate Adult fruit fly specimens were collected from host plants in the research region in 2016-2017 years. All collected specimens were identified according to Hendel (1927), White (1988), Freidberg \& Kugler (1989), Merz (1994), Korneyev \& White (1993, 1999), Kütük (2003a, 2006), Korneyev (2003, 2006), Korneyev et al., (2013) and Yaran (2014), and are stored in the Entomology Laboratory of Gaziantep University Department of Biology.

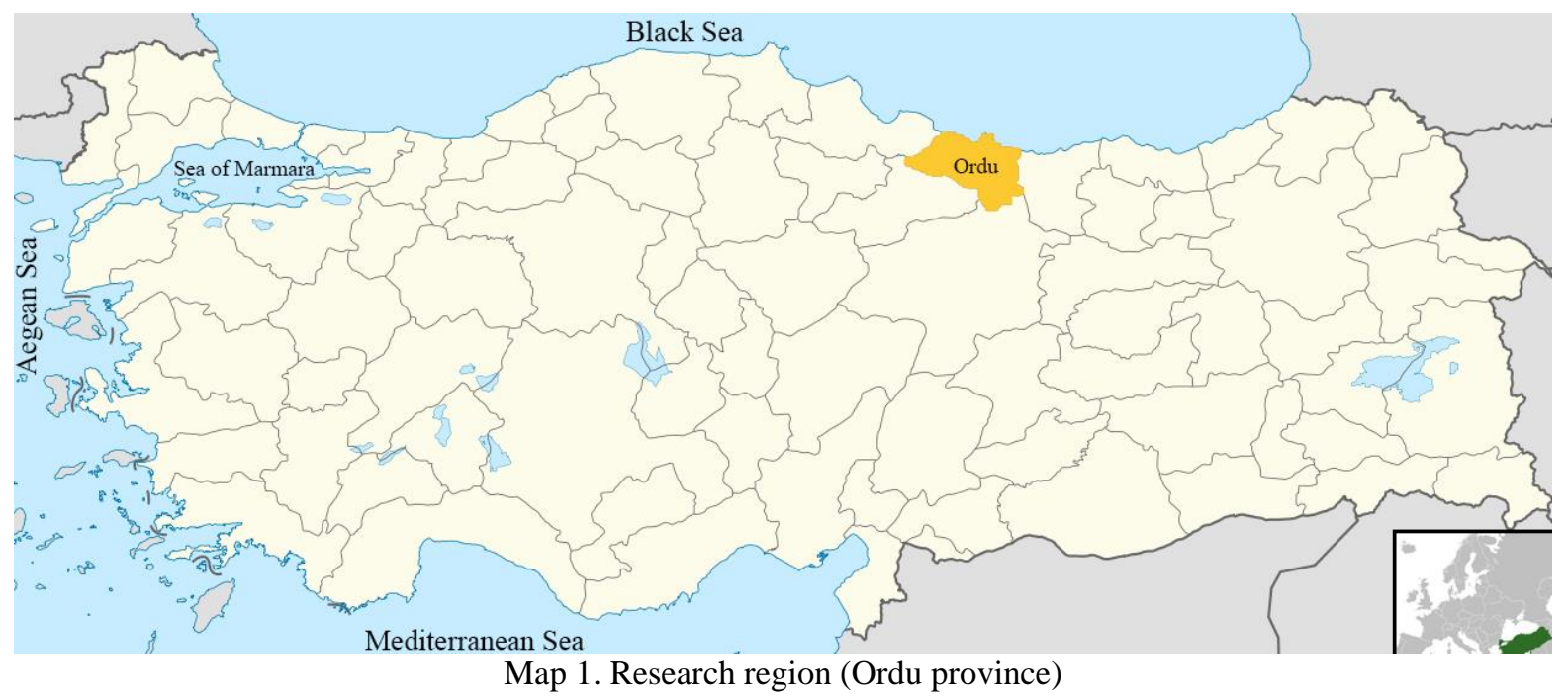

\section{Result}

In this study, fruit fly fauna of Ordu province was determined. Thirty-three species belonging to 11 genera have been identified. Material examined and wing photographs of the species are presented.

The identified species are presented below in alphabetical order.

Acanthiophilus helianthi (Rossi, 1794); (Figure 1).

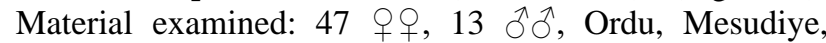
Çaltepe, $40^{\circ} 47^{\prime} \mathrm{N}, 37^{\circ} 72^{\prime} \mathrm{E}, 1061 \mathrm{~m}, 30.06 .2016 ; 1$ ऽे, Görçentepe, Ağıla, 40 46 ${ }^{\prime} \mathrm{N}, 37^{\circ} 36^{\prime} \mathrm{E}, 1158 \mathrm{~m}$,

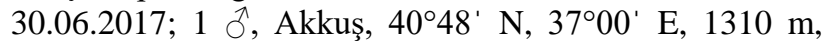

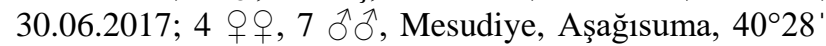
N, 37²4' E, 1430 m, 29.08.2017.
Campiglossa bidentis (Robineau - Desvoidy, 1830); (Figure 2). Material examined: 3 우, $3 \hat{\jmath} \widehat{\jmath}$, Ordu, Ünye, Güzelyalı, $41^{\circ} 08^{\prime} \mathrm{N}, 37^{\circ} 39^{\prime} \mathrm{E}, 70 \mathrm{~m}, 29.06 .2016$; 1 ㅇ, Ulubay, Akpinar, $40^{\circ} 54^{\prime}$ N, 3747' E, 345 m, 30.06.2017.

Campiglossa producta (Loew, 1844); (Figure 3). Material examined: $2 \widehat{\jmath}$, Ordu, Gölköy, Döşek, 4045' N, 37³7' E, 1129 m, 30.06.2017; 1 o, 1 ô, Görçentepe,

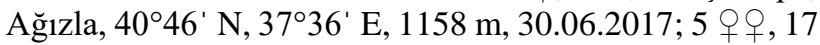

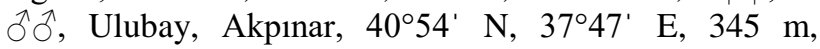
30.06.2017; $1 \mathrm{\delta}$, Karaca, 41 $00^{\prime} \mathrm{N}, 37^{\circ} 37^{\prime} \mathrm{E}, 48 \mathrm{~m}$,

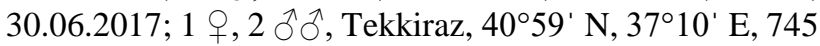
$\mathrm{m}, 30.06 .2017$. 
Chaetorellia carthami Stackelberg, 1929; (Figure 4). Material examined: 1 , Ordu, Akkuş, Kılcanlı, $40^{\circ} 45^{\prime} \mathrm{N}$,

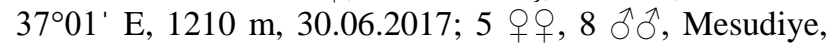
Aşağısuma, $40^{\circ} 28^{\prime} \mathrm{N}, 37^{\circ} 42^{\prime} \mathrm{E}, 1430 \mathrm{~m}, 29.08 .2017$.

Chaetorellia loricata (Rondani, 1830); (Figure 5).

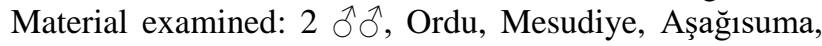
$40^{\circ} 28^{\prime} \mathrm{N}, 37^{\circ} 42^{\prime} \mathrm{E}, 1430 \mathrm{~m}, 29.06 .2017$.

Ensina sonchi (Linnaeus, 1767); (Figure 6). Material examined: 1 \% , Ordu, Ulubay, Akpınar, $40^{\circ} 54^{\prime} \mathrm{N}, 37^{\circ} 47^{\prime}$ E, $345 \mathrm{~m}, 30.06 .2017$.

Spathulina sicula Rondani, 1856; (Figure 7). Material examined: $1 \hat{\sigma}$, Ordu, Mesudiye, Aşağ 1 suma, 40² $28^{\prime} \mathrm{N}$, $37^{\circ} 42^{\prime} \mathrm{E}, 1430 \mathrm{~m}, 29.06 .2017$.

Sphenella marginata (Fallen, 1814); (Figure 8). Material examined: 2 우, $1 \lesssim$, Ordu, Ünye, Güzelyalı, $41^{\circ} 08^{\prime} \mathrm{N}, 37^{\circ} 39^{\prime} \mathrm{E}, 70 \mathrm{~m}, 29.06 .2016 ; 2$ 우우 2 위, Mesudiye, Çaltepe, $40^{\circ} 47^{\prime} \mathrm{N}, 37^{\circ} 72^{\prime} \mathrm{E}, 1061 \mathrm{~m}$,

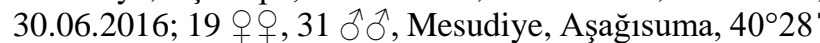
N, 3742' E, 1430 m, 29.06.2017; 1 q, Gölköy, Döşek, $40^{\circ} 45^{\prime} \mathrm{N}, 37^{\circ} 37^{\prime} \mathrm{E}, 1129 \mathrm{~m}, 30.06 .2017$; 1 9, Görçentepe,

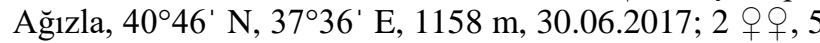

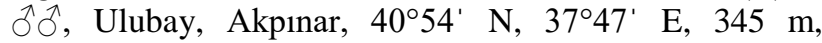

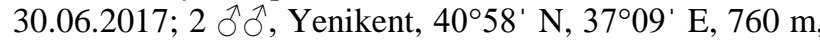
30.06.2017; 1 ô, İkizce, Dumantepe, $40^{\circ} 51^{\prime} \mathrm{N}, 37^{\circ} 01^{\prime} \mathrm{E}$, $1270 \mathrm{~m}, 30.06 .2017$; 2 우, 2 ठิ $\widehat{\partial}$, Mesudiye, Sarıca, $40^{\circ} 42^{\prime} \mathrm{N}, 37^{\circ} 79^{\prime} \mathrm{E}, 1530 \mathrm{~m}, 31.07 .2017$.

Tephritis cometa (Loew, 1840); (Figure 9). Material examined: 2 우, 1 $\mathrm{O}^{\top}$, Ordu, Mesudiye, Çaltepe, $40^{\circ} 47^{\prime} \mathrm{N}$,

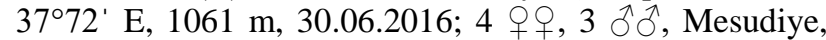
Aşağısuma, $40^{\circ} 28^{\prime} \mathrm{N}, 37^{\circ} 42^{\prime} \mathrm{E}, 1430 \mathrm{~m}, 29.08 .2017$.

Tephritis dioscurea (Loew, 1856); (Figure 10). Material examined: 3 우, $3 \hat{\jmath} \widehat{\jmath}$, Ordu, Mesudiye, Çaltepe, 4047' N, 3772' E, 1061 m, 30.06.2016; 1 , Gölköy,

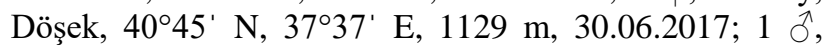
Görçentepe, Ağıla, 4046' N, 37³6' E, $1158 \mathrm{~m}$, 30.06.2017; 5 우우, 4 ํํ․, Mesudiye, Sarica, $40^{\circ} 42^{\prime} \mathrm{N}$,

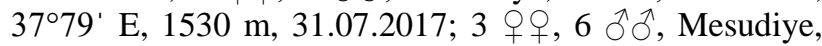
Aşağısuma, $40^{\circ} 28^{\prime} \mathrm{N}, 37^{\circ} 42^{\prime} \mathrm{E}, 1430 \mathrm{~m}, 29.08 .2017$.

Tephritis divisa Rondani, 1871; (Figure 11). Material examined: $1 \delta^{\top}$, Ordu, Ulubay, Akpinar, $40^{\circ} 54^{\prime} \mathrm{N}, 37^{\circ} 47^{\prime}$ E, 345 m, 30.06.2017; 1 o', Tekkiraz, 40 $59^{\prime} \mathrm{N}, 37^{\circ} 10^{\prime} \mathrm{E}$,

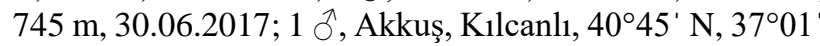

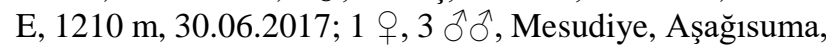
$40^{\circ} 28^{\prime} \mathrm{N}, 37^{\circ} 42^{\prime} \mathrm{E}, 1430 \mathrm{~m}, 29.08 .2017$.

Tephritis fallax (Loew, 1844); (Figure 12). Material examined: $1 \delta^{\top}$, Ordu, Akkuş, 4048' N, 3700' E, $1310 \mathrm{~m}$, 30.06.2017.

Tephritis formosa (Loew, 1844); (Figure 13). Material

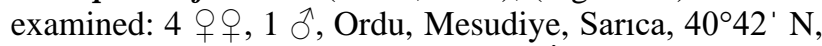

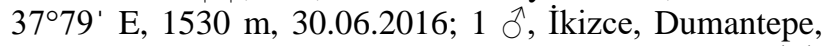
$40^{\circ} 51^{\prime} \mathrm{N}, 37^{\circ} 01^{\prime} \mathrm{E}, 1270 \mathrm{~m}, 30.06 .2017 ; 12$ 우, 22 췻, Mesudiye, Aşağısuma, 40⒉ $8^{\prime} \mathrm{N}, 37^{\circ} 42^{\prime} \mathrm{E}, 1430 \mathrm{~m}$,

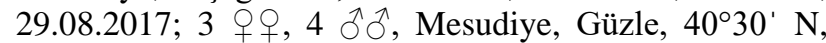
3739' E, 1540 m, 30.08.2017; 1 ㅇ, Mesudiye, Gölköy, $40^{\circ} 36^{\prime} \mathrm{N}, 37^{\circ} 37^{\prime}$ E, $1580 \mathrm{~m}, 31.08 .2017$.
Tephritis hurvitzi Freidberg, 1981; (Figure 14). Material examined: $1{ }^{\top}$, Ordu, Tekkiraz, 40 $59^{\prime} \mathrm{N}, 37^{\circ} 10^{\prime}$ E, $745 \mathrm{~m}, 30.06 .2017$.

Tephritis matricariae (Loew, 1844); (Figure 15). Material examined: $1 \delta^{\top}$, Ordu, Akkuş, $40^{\circ} 48^{\prime} \mathrm{N}, 37^{\circ} 00^{\prime} \mathrm{E}$, $1310 \mathrm{~m}, 30.06 .2017$.

Tephritis nigricauda (Loew, 1856); (Figure 16). Material examined: 1 , , Ordu, Karaca, $41^{\circ} 00^{\prime} \mathrm{N}, 37^{\circ} 37^{\prime}$ E, $48 \mathrm{~m}, 30.06 .2017$.

Tephritis postica (Loew, 1844); (Figure 17). Material examined: 9 qq, $8 \hat{\jmath} \widehat{\jmath}$, Ordu, Mesudiye, Aşağısuma, $40^{\circ} 28^{\prime} \mathrm{N}, 37^{\circ} 42^{\prime} \mathrm{E}, 1430 \mathrm{~m}, 29.06 .2017 ; 1$ ô, Ulubay, Akpınar, 405 $54^{\prime} \mathrm{N}, 37^{\circ} 47^{\prime} \mathrm{E}, 345 \mathrm{~m}, 30.06 .2017$; 5 웅, 5

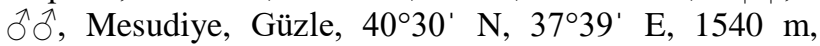

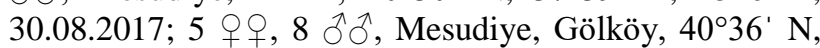
$37^{\circ} 37^{\prime} \mathrm{E}, 1580 \mathrm{~m}, 31.08 .2017$.

Tephritis sauterina Merz, 1994; (Figure 18). Material examined: 5 우, $6 \widehat{\jmath} \hat{\jmath}$, Ordu, Mesudiye, Aşağısuma, $40^{\circ} 28^{\prime} \mathrm{N}, 37^{\circ} 42^{\prime} \mathrm{E}, 1430 \mathrm{~m}, 29.06 .2017$.

Tephritis separata Rondani, 1871; (Figure 19). Material examined: 3 $\circ$, $2 \hat{\delta} \hat{\jmath}$, Ordu, Mesudiye, Aşağısuma, $40^{\circ} 28^{\prime} \mathrm{N}, 37^{\circ} 42^{\prime} \mathrm{E}, 1430 \mathrm{~m}, 29.08 .2017$.

Tephritis vespertina (Loew, 1844); (Figure 20). Material examined: 1 , , Ordu, Ulubay, Akpınar, $40^{\circ} 54^{\prime} \mathrm{N}, 37^{\circ} 47^{\prime} \mathrm{E}, 345 \mathrm{~m}, 30.06 .2017 ; 2$ 우, , Tekkiraz, $40^{\circ} 59^{\prime} \mathrm{N}, 37^{\circ} 10^{\prime} \mathrm{E}, 745 \mathrm{~m}, 30.06 .2017$.

Terellia gynaecochroma (Hering, 1937); (Figure 21).

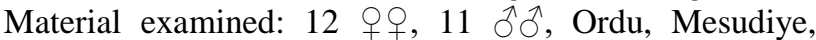
Aşağısuma, $40^{\circ} 28^{\prime} \mathrm{N}, 37^{\circ} 42^{\prime} \mathrm{E}, 1430 \mathrm{~m}, 29.06 .2017$.

Terellia luteola (Wiedemann, 1830); (Figure 22). Material examined: 2 오, $2 \hat{\jmath} \hat{o}$, Ordu, Mesudiye, Aşağısuma, $40^{\circ} 28^{\prime} \mathrm{N}, 37^{\circ} 42^{\prime} \mathrm{E}, 1430 \mathrm{~m}, 29.08 .2017$.

Terellia ruficauda (Fabricius, 1794); (Figure 23). Material examined: 1 을, Ordu, İkizce, Dumantepe, $40^{\circ} 51^{\prime}$ $\mathrm{N}, 37^{\circ} 01^{\prime} \mathrm{E}, 1270 \mathrm{~m}, 30.06 .2017$.

Terellia serratulae (Linnaeus, 1758); (Figure 24). Material examined: 1 \% , Ordu, Akkuş, $40^{\circ} 48^{\prime} \mathrm{N}, 37^{\circ} 00^{\prime} \mathrm{E}$, $1310 \mathrm{~m}, 30.06 .2017$; 5 우, 5 $\widehat{\jmath}$, Mesudiye, Aşağısuma, $40^{\circ} 28^{\prime} \mathrm{N}, 37^{\circ} 42^{\prime} \mathrm{E}, 1430 \mathrm{~m}, 29.08 .2017$.

Terellia tussilaginis (Fabricius, 1775); (Figure 25).

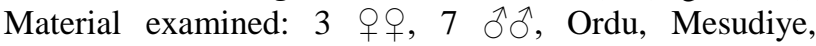
Aşağısuma, $40^{\circ} 28^{\prime} \mathrm{N}, 37^{\circ} 42^{\prime} \mathrm{E}, 1430 \mathrm{~m}, 29.08 .2017$.

Trupanea amoena (Frauenfeld, 1857); (Figure 26). Material examined: 1 ${ }^{\circ}$, Ordu, Tekkiraz, $40^{\circ} 59^{\prime} \mathrm{N}, 37^{\circ} 10^{\prime}$ E, $745 \mathrm{~m}$, 30.06.2017.

Trupanea stellata (Fuesslin, 1775); (Figure 27). Material examined: 1 9, Ordu, Ulubay, Akpınar, 40 $54^{\circ} \mathrm{N}$, $37^{\circ} 47^{\prime} \mathrm{E}, 345 \mathrm{~m}, 30.06 .2017$; 2 우, Tekkiraz, 405' $\mathrm{N}$, $37^{\circ} 10^{\prime} \mathrm{E}, 745 \mathrm{~m}, 30.06 .2017$. 
Urophora affinis (Frauenfeld, 1857); (Figure 28), Material examined: 3 우, 2 $\delta^{\lambda}$, Ordu, Mesudiye, Güzle,

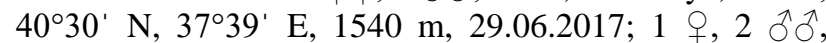
Mesudiye, Gölköy, 40³6' N, 37³7' E, $1580 \mathrm{~m}$,

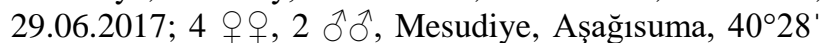
$\mathrm{N}, 37^{\circ} 42^{\prime} \mathrm{E}, 1430 \mathrm{~m}, 29.08 .2017$.

Urophora jaceana (Hering, 1935); (Figure 29).

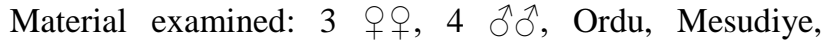

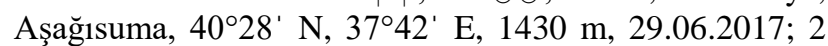

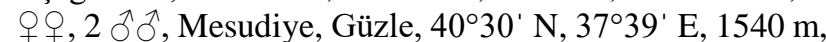
29.06.2017; 1 ㅇ, 1 §̊’, Mesudiye, Gölköy, 40³6' N, 37³7 E, $1580 \mathrm{~m}, 29.06 .2017$.

Urophora mauritanica Macquart 1851; (Figure 30). Material examined: 15 우, 11 ふぇ, Ordu, Mesudiye, Aşağısuma, 40⒉ $8^{\prime} \mathrm{N}, 37^{\circ} 42^{\prime} \mathrm{E}, 1430 \mathrm{~m}$, 29.06.2017.
Urophora solstitialis (Linnaeus, 1758); (Figure 31). Material examined: 4 ${ }^{\circ}, 9 \hat{\jmath} \widehat{\partial}$, Ordu, Mesudiye, Aşağısuma, $40^{\circ} 28^{\prime} \mathrm{N}, 37^{\circ} 42^{\prime} \mathrm{E}, 1430 \mathrm{~m}, 29.06 .2017$.

Urophora stylata (Fabricius, 1775); (Figure 32). Material examined: 3 우, 5 ऽิ่, Ordu, Ünye, Güzelyalı, $41^{\circ} 08^{\prime} \mathrm{N}$,

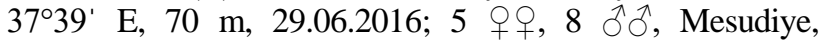
Aşağısuma, $40^{\circ} 28^{\prime} \mathrm{N}, 37^{\circ} 42^{\prime} \mathrm{E}, 1430 \mathrm{~m}, 29.06 .2017$; 3 우우,

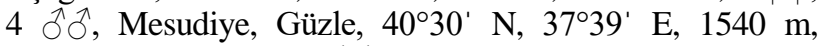

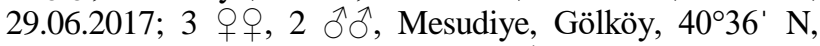
$37^{\circ} 37^{\prime} \mathrm{E}, 1580 \mathrm{~m}, 29.06 .2017 ; 1$ ㅇ, 1 ò , Tekkiraz, 405' $\mathrm{N}$, $37^{\circ} 10^{\prime} \mathrm{E}, 745 \mathrm{~m}, 30.06 .2017 ; 1$ ㅇ, $2 \mathrm{o}^{\lambda} \mathrm{o}^{\mathrm{N}}$, İkizce, Dumantepe, $40^{\circ} 51^{\prime} \mathrm{N}, 37^{\circ} 01^{\prime} \mathrm{E}, 1270 \mathrm{~m}, 30.06 .2017$.

Xyphosia miliaria (Schrank, 1781); (Figure 33). Material examined: 1 9 , Ordu, İkizce, Dumantepe, $40^{\circ} 51^{\prime}$ $\mathrm{N}, 37^{\circ} 01^{\prime} \mathrm{E}, 1270 \mathrm{~m}, 30.06 .2017 ; 1$ ô, Akkuş, 4048' N, $37^{\circ} 00^{\prime} \mathrm{E}, 1310 \mathrm{~m}, 30.06 .2017$.

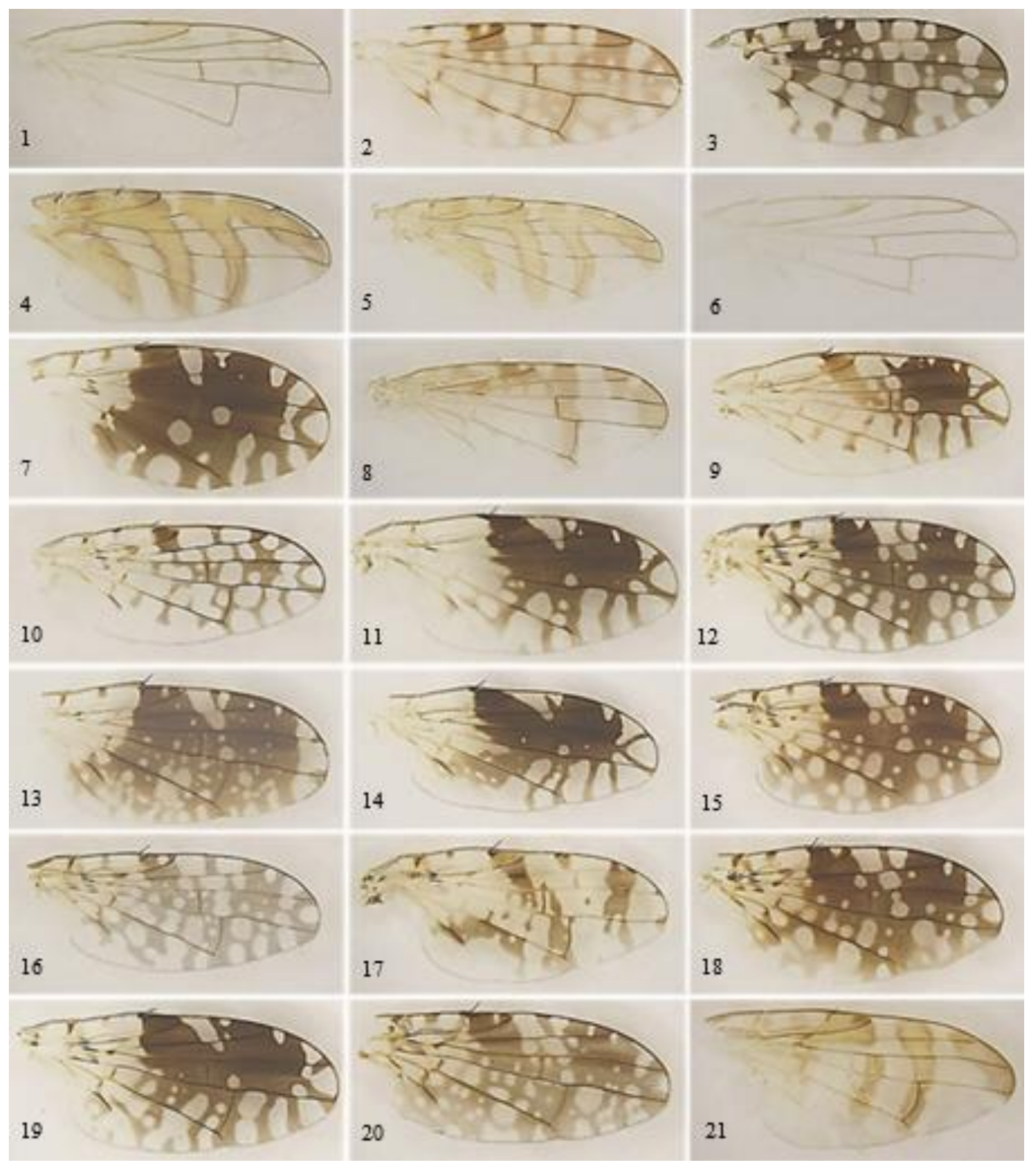

Figures 1-21. Wings of fruit fly species; 1. Acanthiophilus helianthi, 2. Campiglossa bidentis, 3. Cam. producta, 4. Chaetorellia carthami, 5. Cha. loricata, 6. Ensina sonchi, 7. Spathulina sicula, 8. Sphenella marginata, 9. Tephritis cometa, 10. Tep. dioscurea, 11. Tep. divisa, 12. Tep. fallax, 13. Tep. formosa, 14. Tep. hurtvitzi, 15. Tep. matricariae,

16. Tep. nigricauda, 17. Tep. postica, 18. Tep. sauterina, 19. Tep. separata, 20. Tep. vespertina, 21. Terellia gynaecochroma. 


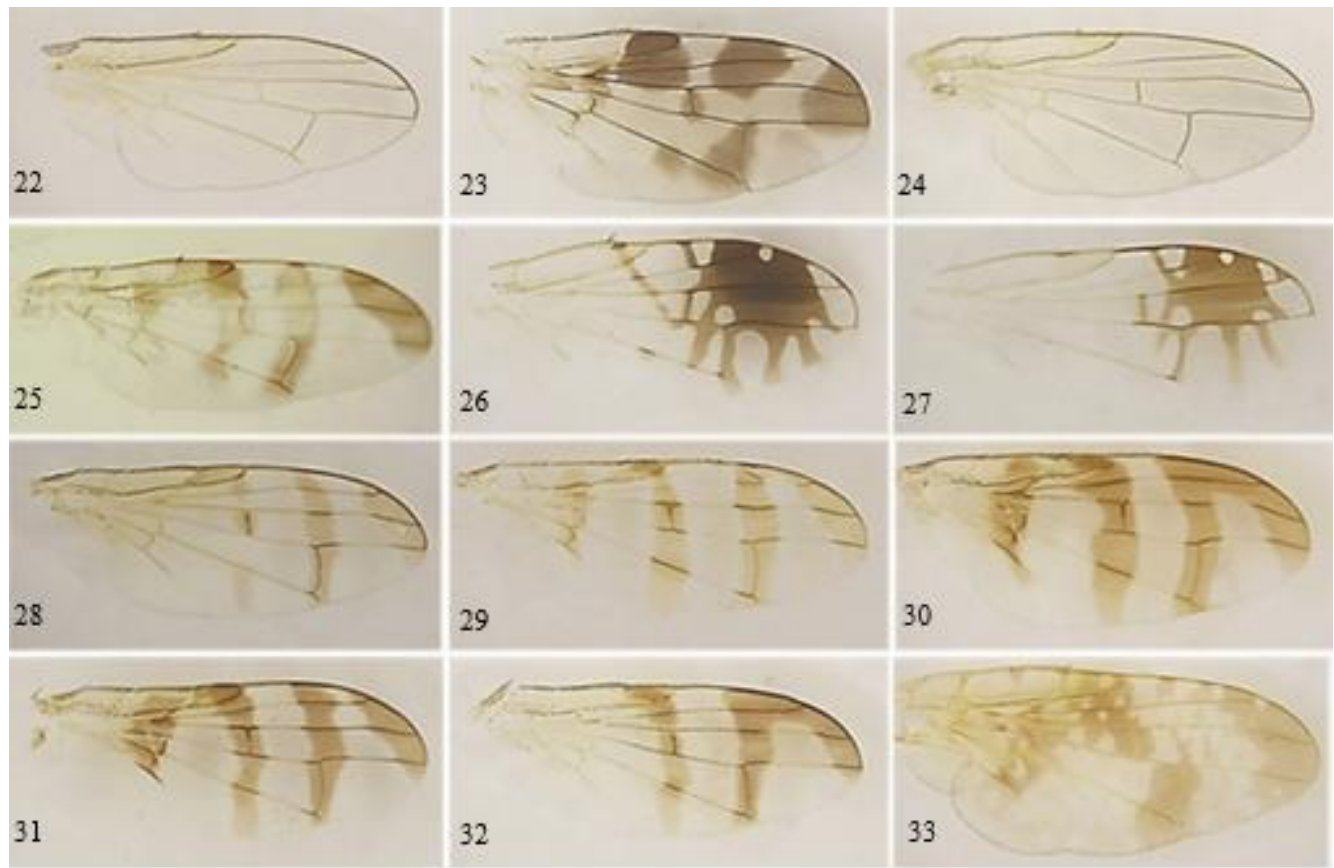

Figures 22-33. Wings of fruit fly species; 22. Terellia luteola, 23. Ter. ruficauda, 24. Ter. serratulae, 25. Ter. tussilaginis, 26. Trupanea amoena, 27. Tru. stellata, 28. Urophora affinis, 29. Uro. jaceana, 30. Uro. mauritanica, 31. Uro. solstitialis, 32. Uro. stylata, 33. Xyphosia miliaria.

\section{Discussion}

In this study, adult fruit fly specimens were collected from host plants in the research region between 2016-2017 years and were diagnosed. As a result, thirty-three species belonging to 11 genera were determined from Ordu province. There has been no previous study on fruit fly fauna in the research region. For this reason, all of the identified species have been reported for the first time in Ordu province.

Ordu province is located in the Black Sea Region of Turkey. The entire province is under the influence of the Black Sea climate. The area along the Black Sea coast in the north is quite poor in terms of plant diversity. Other areas are covered with dense forests. The vegetation cover of the region is limited to the species specific to the Black Sea climate. This situation has also limited the diversity of fruit flies.

The result of this study is in line with similar studies conducted before. The data obtained make significant contributions to the fruit fly fauna of Turkey. It will also be a resource for future studies.

\section{References}

Bayrak N. 2011. Kuzeydoğu Anadolu Bölgesi Tephritinae ve Terellinae (Diptera: Tephritidae) Türleri Üzerinde Faunistik ve Sistematik Çalı̧̧malar. PhD Thesis, Atatürk University, Institute of Sciences, Erzurum, Turkey.

Çalışkan Keçe AF, Çatal BÖ, Ulusoy MR. 2019. A new invasive species in Turkey: Dacus ciliatus Loew, 1862 (Diptera: Tephritidae). Turkish Journal of Entomology, 43(1): 25-30.

Freidberg A, Kugler J. 1989. Fauna Palaestina Insecta IV. Diptera: Tephritidae. Israel Academy of Sciences and Humanities.

Freidberg A, Kütük M. 2002. A new species of Tephritis from Turkey, with a key to the species of the Tephritis pulchra group. Israel Journal of Zoology, 48: 295-311.
Freidberg A. 2016. New taxa of Carpomyini, with special emphasis on Goniglossum (Diptera: Tephritidae: Trypetinae). Zootaxa, 4144(1): 054-070.

Görmez V, Kütük M. 2020. Fruit fly (Diptera: Tephritidae) fauna of Çorum and Sinop provinces with two new records for Turkey. Turkish Journal of Entomology 44(1): 23-38.

Görmez V. 2011. Kahramanmaraş İlinde Meyve Sinekleri (Diptera: Tephritidae) Faunası ve Sistematiği Üzerine Araştırmalar. MSc Thesis, Gaziantep University, Institute of Sciences, Gaziantep, Turkey.

Han HY, Kütük M. 2006. A new species of Myoleja Rondani (Diptera: Tephritidae) from Turkey with a key to the known species of the genus. Zootaxa. 1155: 25-33.

Hendel F. 1927. 49. Trypetidae die Fliegen der Palaerktischen Region. Book on Demand. ASIN: B0068PDJF4.

Korneyev VA, Evstigneev DA, Karimpour Y, Kütük M, Mohamadzade Namin S, Koyuncu MÖ. Yaran M. 2013. Revision of the Terellia virens group (Diptera, Tephritidae) with description of three new species. Vestnik Zoologii, 47(1): 3-25.

Korneyev VA, White IM. 1993. Fruit-flies of the Eastern Palaearctic species of Urophora R.-D. (Diptera: Tephritidae). II. Review of species of the subgenus Urophora s. str. (Communication 2). Entomologicheskoe obozrenie, 72(1): 232-247.

Korneyev VA, White IM. 1999. Fruit flies of genus Urophora R-D. (Diptera: Tephritidae) of east palaerctic. III. key to species. Entomologicheskoe Obozrenie, 78(2): 464-482.

Korneyev VA. 2003. New and Little-Known Tephritidae (Diptera, Cyclorrhapha) from Europe. Vestnik Zoologii. 37(3): 3-12.

Korneyev VA. 2006. A revision of the quadratula group of the genus Terellia Robineau-Desvoidy (Diptera: Tephritidae). In: Freidberg A., ed. Biotaxonomy of Tephritoidea: Israel Journal of Zoology, 35-36: 341-366.

Kütük M, Bayrak N, Hayat R. 2011. A new species of Terellia (Diptera: Tephritidae) from Turkey with a key to Turkish species of the subgenus Cerajocera. Turkish Journal of Entomology, 35(2): 207-213. 
Kütük M, Bayrak N, Hayat R. 2012. A new species of Tephritis Latreille (Diptera: Tephritidae) from Turkey. Turkish Journal of Zoology, 36(4): 475-480.

Kütük M, Özaslan M. 2006. Faunistical and Systematical Studies on the Trypetınae (Diptera: Tephritidae) in the Turkey along with a New Record to Turkish Fauna. Munis Entomology \& Zoology, 1(2): 173-178.

Kütük M, Özgür AF. 2003a. Faunistical and systematical studies on the genus Tephritis Latreille, 1804 (Diptera: Tephritidae) in the South West of Turkey along with new records. Turkish Journal of Entomology, 27(4): 243-252.

Kütük M, Özgür AF. 2003b. Türkiye için 8 Yeni Kayıt ve Güney Batı Anadolu Bölgesinde Terellinae (Tephritidae: Diptera) Faunası ve Sistematiği Üzerine Araştırmalar. Journal of Agriculture Faculty, Çukurova University, 18(3): 105-114.

Kütük M, Özgür AF. 2004. Two New Records of the Fruit Flies (Diptera: Tephritidae) for Fauna of Turkey. Journal of Agriculture Faculty, Cukurova University, 19(1): 53-56.

Kütük M, Varol Mİ. 2006. Records of Two Species of Terellia Ronineau-Desvoidy, 1830 (Diptera: Tephritidae) New to the Turkish Fauna. Turkish Journal of Zoology, 30: 99-102.

Kütük M, Yaran M, Hayat R, Koyuncu MÖ, Görmez V, Aytekin HU. 2013. The determination of fruit fly (Diptera: Tephritidae) fauna in Adiyaman, Kilis, and Şanlıurfa provinces with a new record for Turkish fauna. Turkish Journal of Zoology, 37: 38-49.

Kütük M, Yaran M. 2020. A new species and a new record of Tephritis Latreille, 1804 (Diptera: Tephritidae) from Turkey. Turkish Journal of Entomology, 22(2):215-222.

Kütük M. 2003a. Güneybatı Anadolu Bölgesi Tephritidae Faunası ve Sistematiği Üzerine Araştırmalar. PhD Thesis, Cukurova University, Institute of Sciences, Adana, Turkey.

Kütük M. 2003b. Doğu Akdeniz Bölgesi Urophora RobineauDesvoidy (Diptera: Tephritidae) faunası ve sistematiği üzerine araştırmalar. Turkish Journal of Entomology, 27(2): 149-160.

Kütük M. 2005. Güney Batı Anadolu Bölgesinden Saptanan Türkiye İçin Yeni Kayıt Meyve Sineği (Tephritidae: Diptera) Türleri. Journal of Agriculture Faculty, Çukurova University, 20(4): 29-36.

Kütük M. 2006. A New Species of Urophora RobineauDesvoidiy, 1830 (Diptera: Tephritidae) from Turkey, with a Key to the Species of the Urophora guadrifasciata Group. Turkish Journal of Zoology, 30: 147-153.

Kütük M. 2008. A new species of Tephritis Latreille (Diptera: Tephritidae) from Turkey. Belgian Journal of Zoology, 138(2): 132-134.

Kütük M. 2009a. A New Species of Urophora RobineauDesvoidy, 1830 (Diptera: Tephritidae) from Turkey. Entomological News, 120(1): 101-105.
Kütük M. 2009b. A new species of Terellia Robineau-Desvoidy (Diptera: Tephritidae) from Turkey. Turkish Journal of Zoology, 33: 297-300.

Li Z. Jiang F, Ma X, Fang Y, Sun Z, Qin Y, Wang Q. 2013. Review on prevention and control techniques of Tephritidae invasion. Plant Quarantine, 27: 1-10.

Merz B. 1994. Insecta Helvetica fauna, 10. Diptera, Tephritidae. Centre Suisse de Cartographie de la Faune (CSCF), Neuchatel.

Özgür AF, Kütük M. 2003. Adana İli Meyve Sinekleri (Tephritidae: Diptera) Faunasının Tespiti. Journal of Agriculture Faculty, Çukurova University, 18(2): 35-44.

Pakyürek B. 2006. Gazi Üniversitesi Zooloji Müzesindeki Tephritidae (Diptera) familyası örneklerinin değerlendirilmesi. MSc Thesis, Gazi University, Institute of Sciences, Ankara, Turkey.

Pape T, Vladimir B, Mikhail BM. 2011. Order Diptera Linnaeus, 1758. In: Zhang, Z. - Q. (Ed.) Animal biodiversity: An outline of higher - level classification and survey of taxonomic richness. Zootaxa, 3148: 222-229.

White IM. 1988. Tephritid flies (Diptera: Tephritidae). Handbook for the identification of British insects. Intl Specialized Book Services. ISBN-10: 0901546682.

Yaran M, Görmez V. 2020. A new species of Tephritis Latreille, 1804 (Diptera: Tephritidae) from Turkey. Zootaxa 4838(2):283-288

Yaran M, Kütük M, Görmez V, Koyuncu MÖ. 2018a. A new species and additional record of Terellia Robineau- Desvoidy (Diptera: Tephritidae) from Turkey with a key for the Cerajocera group. Turkish Journal of Zoology, 42: 661-665.

Yaran M, Kütük M, Görmez V, Koyuncu MÖ. 2018b. Some additional notes on fruit fly (Diptera: Tephritidae) fauna and a new genera and species record from Turkey. Biological Diversity and Conservation, 11(3): 141-144.

Yaran M, Kütük M. 2014. A new species of Urophora RobineauDesvoidy, 1830 (Diptera: Tephritidae) from Turkey with a key to the dzieduszyckii group. Turkish Journal of Entomology, 38: 149-155.

Yaran M, Kütük M. 2015. Two new records of fruit flies (Diptera: Tephritidae) with fauna of Aksaray and Mersin provinces. Turkish Journal of Zoology, 39: 1056-1070.

Yaran M, Kütük M. 2016. Fruit flies (Diptera: Tephritidae) fauna in Nevşehir and Niğde provinces with a new record from Turkey. Turkish Journal of Zoology, 40(5): 785-800.

Yaran M. 2014. Aksaray, Mersin, Nevșehir ve Niğde illerinde meyve sinekleri (Diptera: Tephritidae) faunası ve sistematiği üzerine araştırmalar. $\mathrm{PhD}$ Thesis, Gaziantep University, Institute of Sciences, Gaziantep, Turkey. 